\title{
The use of circulating cathodic antigen rapid test and serology for diagnosis of active Schistosoma mansoni infection in migrants in Italy, a non-endemic country: a cross sectional study
}

\author{
Laura Infurnari ${ }^{1}$, Laura Galli ${ }^{2}$, Alba Bigoloni ${ }^{2}$, Alessia Carbone ${ }^{2}$, Stefania Chiappetta ${ }^{2}$, Angelo Sala ${ }^{3}$, \\ Norberto Ceserani ${ }^{2}$, Adriano Lazzarin ${ }^{2,4}$, Antonella Castagna ${ }^{2,4}$, Giovanni Gaiera ${ }^{2 /+}$
}

${ }^{1}$ San Raffaele Scientific Institute, Laboratory of Microbiology and Virology, Milan, Italy

${ }^{2}$ San Raffaele Scientific Institute, Infectious Diseases Department, Milan, Italy

${ }^{3}$ Saint Michel Centre, Society of Priests of the Sacred Heart of Betharram, Health Pastoral Diocese of Bouar, Bouar, Central African Republic

${ }^{4}$ Università Vita-Salute San Raffaele, Milan, Italy

Diagnosis of schistosomiasis in migrants coming from endemic areas can be difficult, especially in asymptomatic subjects. Light-intensity disease, in fact, may be missed due to the low sensitivity of the stool microscopy and serologic testing cannot distinguish between a resolved infection and an active infection in patients who have been infected and treated in the past, because specific antibodies can persist despite cure. We describe a cross-sectional study conducted on 82 migrants tested for Schistosoma mansoni on single blood (anti-schistosome antibodies, total $\mathrm{IgE}$ ) and urine [point-of-care (POC) circulating-cathodic-antigen (CCA) test] samples. A positive POC-CCA test (active infection) resulted in two untreated patients with a positive serology while all patients $(\mathrm{n}=66)$ with a past infection showed a negative POC-CCA test. POC-CCA urine test in combination with serology may be helpful in rapidly differentiate active from past $S$. mansoni infection in migrants coming from endemic areas.

Key words: diagnosis - infectious diseases - international health - schistosomiasis - travel medicine - tropical medicine

Schistosoma mansoni is a parasite endemic in large parts of Africa (Hotez et al. 2014) and the reference standard for its diagnosis is stool microscopy (Kato-Katz or Ritchie tests) (Katz et al. 1972, Clerinx \& van Gompel 2011, Gomes et al. 2014). The sensitivity of stool microscopy is, however, limited because of the number of eggs contained in each stool sample that can be reduced in light-infections $(<100$ eggs/gram stool) and that frequently change on a day-to-day basis; moreover, this test depends on operator-experience (Navaratnam et al. 2012, Utzinger et al. 2012, Erko et al. 2013, Ochodo et al. 2015). Usually, adult migrants with chronic S. mansoni infections have light-intensity disease that may be missed due to the unspecific clinical presentation, the small number of eliminated eggs and the low sensitivity of the most widely used diagnostic assays (Utzinger et al. 2012). Then, there is the need for more sensitive, user-friendly and rapid diagnostic tests. Many studies evaluated the applicability of circulating cathodic antigen (CCA) test for the diagnosis of S. haematobium with very low sensitivity and unsatisfactory results (Obeng et al. 2008, Midzi et al. 2009, Stothard et al. 2009). On the contrary, the use of CCA test in S. mansoni infections was highly sensitive, rapid and non-invasive. In particular, various studies conducted in endemic areas

doi: 10.1590/0074-02760160355

+ Corresponding author: gaiera.giovanni@hsr.it

Received 7 August 2016

Accepted 4 March 2017 comparing the use of CCA test on urine to Kato-Katz technique on stools demonstrated a superior sensitivity of CCA test in detecting S. mansoni infections (Coulibaly et al. 2011, 2013, Shane et al. 2011, Navaratnam et al. 2012, Tchuenté et al. 2012, Colley et al. 2013, Erko et al. 2013, Adriko et al. 2014). According to the Ochodo et al.'s review (Ochodo et al. 2015), the sensitivity of CCA test was $89 \%$ (ranging from $62-97 \%$ ) and the specificity was $55 \%$ (ranging from $27-84 \%$ ); authors interpreted the values of specificity as the misdiagnosed infections of patients with light-infections ( $<100$ eggs/gram stool). The lack of specificity can be increased to $76 \%$ if CCA is combined with Kato-Katz (Ruganuza et al. 2015) or if CCA test is repeated on multiple urine samples (Erko et al. 2013). Sensitivity seems to be higher in lower transmission setting (Sousa-Figueiredo et al. 2013).

In western countries, the use of point-of-care-CCA (POC-CCA) is not yet standardised, even if there is some new example in the recent literature: Becker et al. (2015) described two cases in which CCA urine test was used to confirm diagnosis of $S$. mansoni infection in symptomatic subjects.

Here, we report the results of the application of a urine-based antigen detection test for rapid, non-invasive diagnosis of $S$. mansoni infection in asymptomatic migrants coming from endemic areas.

This is a cross-sectional study on 82 asymptomatic migrants, living in Italy and coming from Egypt, who participated to a survey for the assessment of schistosomiasis at the out clinic of Tropical Medicine of the Infectious Diseases Department, San Raffaele Hospital, since February 2014 to January 2016. 
TABLE

Patients characteristics according to serology for Schistosoma spp

\begin{tabular}{|c|c|c|}
\hline Characteristic & $\begin{array}{l}\text { Anti-schistosome antibodies } \\
\text { Negative }(\mathrm{n}=23)\end{array}$ & $\begin{array}{l}\text { Anti-schistosome antibodies } \\
\text { Positive }(\mathrm{n}=59)\end{array}$ \\
\hline Median age, years (IQR) & $47.6(34.1-55.5)$ & $43.7(37.4-48.8)$ \\
\hline Male gender, $\mathrm{n}(\%)$ & $22(96 \%)$ & $57(97 \%)$ \\
\hline \multicolumn{3}{|l|}{ Nationality } \\
\hline Egyptian & $22(96 \%)$ & $59(100 \%)$ \\
\hline Median length of residence in Italy, years (IQR) & $15(11.5-24.5)$ & $17(14-22)$ \\
\hline \multicolumn{3}{|l|}{ Travel in endemic area, $\mathrm{n}(\%)$} \\
\hline$\leq 12$ months before study enrolment & $4(50 \%)$ & $13(77 \%)$ \\
\hline$>12$ months before study enrolment & $4(50 \%)$ & $3(23 \%)$ \\
\hline Median IgE, UI/mL (IQR) & $34(0-166)$ & $179(49-571)$ \\
\hline Elevated $(>100 \mathrm{UI} / \mathrm{mL})$ & $7(30 \%)$ & $38(68 \%)$ \\
\hline \multicolumn{3}{|l|}{ Urine POC-CCA test, $\mathrm{n}(\%)$} \\
\hline Negative & $23(100 \%)$ & $57(97 \%)$ \\
\hline Positive & 0 & $2(3 \%)$ \\
\hline Treatment for S. mansoni in the past, n (\%) & $9(39 \%)$ & $46(78 \%)$ \\
\hline Months since last treatment & $54.5(33.0-89.2)$ & $28.5(15.5-56.4)$ \\
\hline
\end{tabular}

IQR: interquartile range; POC-CCA: point-of-care-circulating cathodic antigen.

Subjects attending the Travel and Tropical Medicine Clinic for a post-travel (in an endemic area) visit were informed by a travel doctor about the purpose and procedures of this study; participation was voluntary and patients interested in participating the study provided their oral consent. All patients participating to the study were informed about the results of the serological and POCCCA assessments. All patients with an active $S$. mansoni infection were treated with praziquantel $(40 \mathrm{mg} / \mathrm{kg} / \mathrm{day}$ in two doses for three days).

During consultation, patients were screened for the determination of anti-schistosome antibodies, by performing an enzyme immunoassay that uses purified $S$. mansoni antigen (NovaLisa Schistosoma mansoni IgG ELISA, NovaTec Immunodiagnostica $\mathrm{GmbH}$ ), and total IgE; a single urine sample was collected and tested by using a POC assay to detect CCA for the diagnosis of S. mansoni infection (A\&B Rapid Test Schistosomiasis CCA, Lucca Italy). A drop of urine and a drop of reagent were placed on a POC-CCA cassette; the results were read after $20 \mathrm{~min}$ and recorded as either negative, trace or positive according to the development of a coloured test band; a second band (control line) had to show up to make sure that the test was valid.

Results were scored as: negative if the control line developed but no pink test line appeared; trace if a lightpink test line appeared; positive if a dark-pink test line appeared and its colour may be equal or more intense than that of the control line. This classification system is similar to that adopted in other studies (Coulibaly et al. 2011, 2013, Shane et al. 2011, Stothard et al. 2011).

Travels and previous treatments for schistosomiasis were also investigated.

Total $\operatorname{IgE}$ were considered as elevated if values were above the upper normal limit ( $>100 \mathrm{UI} / \mathrm{mL}$ ).
An active infection to be treated was defined in presence of a single trace or positive POC-CCA result associated with positive anti-schistosome antibodies; a past infection with no need of treatment was defined by the presence of a treatment for schistosomiasis in the past or positive anti-schistosome antibodies associated with a single negative POC-CCA test; patients were defined as uninfected if both anti-schistosome antibodies and POC-CCA were negative and if they have never received a previous treatment for schistosomiasis.

The performance of the considered POC-CCA test was also evaluated in five healthy volunteer subjects never exposed to Schistosoma and in 10 symptomatic patients with an active schistosomiasis diagnosed in Saint Michel Centre (Central African Republic) by microscopic stool analysis. Healthy subjects and patients with active S. mansoni infections were assessed by anti-schistosome antibodies, and POC-CCA test as negative and positive controls.

Results were described as median [interquartile range $(\mathrm{IQR})]$ or frequency (\%) in relation to continuous or categorical variables, respectively. Data were analysed by use of the SAS Software, release 9.2 (SAS Institute, Cary, NC).

Overall 82 migrants were evaluated: $95 \%$ males, with a median (IQR) age of 43.8 (37.1-50.7) years. They lived in Italy since a median (IQR) of 17 (14.0-23.0) years and only 24/82 (29\%) had a recent travel in endemic sites. Patients characteristics according to serology for Schistosoma spp are shown in Table.

Two male subjects $(2.4 \%)$ without urinary symptoms had an active infection; 66 subjects (81\%) had a past $S$. mansoni infection, while 14 people had neither an active nor a past infection.

None of the patients with an active infection and $55 / 66$ patients with a past infection (nine patients with a negative serology and a negative POC-CCA test; 46 pa- 
tients with a positive serology and a negative POC-CCA test) had been treated for schistosomiasis in the past; the median (IQR) time since the last previous treatment was of 31.2 (16.6-65.1) months.

Elevated total IgE were observed in one out of two patients with an active $S$. mansoni infection as well as in $41 / 66(62 \%)$ patients with a past infection.

The two patients with an active infection were subsequently treated with praziquantel $(40 \mathrm{mg} / \mathrm{kg} / \mathrm{day}$ in two doses for three days); they were re-assessed after three months from the end of the treatment and a negative POC-CCA test was observed in both cases.

All the five healthy subjects were found to have negative serology and a negative POC-CCA test while positive serology and a positive POC-CCA test was observed in the 10 symptomatic patients with an active schistosomiasis.

Findings of this study seem to suggest that a positive POC-CCA test is likely associated with the presence of an active infection in patients with a positive serology, no previous treatment for schistosomiasis and regardless of total IgE values. In addition, a negative single POCCCA test, regardless of total IgE values, may represent a sign of previous $S$. mansoni infection if associated with a positive serology (immunological memory) or a previous treatment. Such interpretation seems also supported by the good performance of the considered POC-CCA urine test as compared to microscopic examination in a small group of healthy volunteer subjects with negative serology never exposed to Schistosoma and of patients with an active schistosomiasis and positive serology. A negative POC-CCA test was found in all the healthy subjects while all patients with an active infection were CCA-positive.

Migration from Africa into Europe has greatly increased in recent years and European countries have to face with imported infectious diseases not typically present in Europe (de Laval et al. 2014). Serologic testing for anti-schistosome antibodies is indicated for diagnosis of travelers or immigrants from endemic areas who have not been treated for schistosomiasis in the past. In patients who have been infected and treated in the past, serologic testing cannot distinguish resolved infection from active infection because specific antibodies can persist despite cure (CDC 2016). The antigen test can detect active infection and its validity is supported by findings of previous studies showing that the positive and negative predictive values of a single POC-CCA test are $77 \%$ and $72-89 \%$, respectively, if compared to multiple Kato-Katz thick smears as diagnostic reference standard (Erko et al. 2013, Becker et al. 2015).

As a strength of the study, we highlight that, to our knowledge, this is the first study assessing a sample of patients (although limited in number) living in nonendemic countries tested for schistosomiasis because of their origin instead of symptoms (Becker et al. 2015). Among the study limitations, we mention the small number of considered patients, likely affecting the estimates precision; for this reason, our findings may not be intended as confirmatory but rather exploratory. Another limitation is that no stool samples were available in the study patients, impairing the assessment of
POC-CCA performance (i.e., sensitivity, specificity, positive and negative predictive value) as compared to microscopy, still representing the reference standard for schistosomiasis diagnosis. Further studies including this comparative assessment would be of great value.

Epidemiological surveys in large population of migrants to be tested with POC-CCA are warranted in order to prevent $S$. mansoni infection long-term complications and to reduce health care costs avoiding useless treatments in case of past Schistosoma infection (i.e. persistence of positive serology).

In conclusion, the reported data seem to suggest that the use of POC-CCA urine test may be a simple, noninvasive test to differentiate active from past $S$. mansoni infection and therefore rapidly guide treatment initiation in migrants from endemic areas and living in nonendemic countries. Further, larger studies, also providing a comparative assessment with stool microscopy, would certainly be of value in elucidating the potential usefulness and cost-effectiveness of POC-CCA test as a screening diagnostic tool also in asymptomatic migrants living in non-endemic high-income countries.

\section{ACKNOWLEDGEMENTS}

To A\&B Professional (Lucca, Italy), importer of the POC-CCA urine test kits and responsible of their release onto Italian market under the commercial brand A\&B Rapid Test Schistosomiasis CCA.

\section{AUTHORS' CONTRIBUTION}

GG, AnC and AL - Designed the study; GG, AS, SC, AlC, $\mathrm{AB}$ and $\mathrm{NC}$ - participated in the conduction of the study; LG, LI and GG - analysed the data; LG, LI, SC, AlC and GG - contributed to the interpretation of the data; LI and LG - wrote the first draft of the paper; $\mathrm{AnC}, \mathrm{GG}, \mathrm{SC}$ and $\mathrm{AlC}$ - contributed in the writing of the paper. All authors read and approved the final manuscript. GG is guarantor of the paper.

\section{REFERENCES}

Adriko M, Standley CJ, Tinkitina B, Tukahebwa EM, Fenwick A, Fleming FM, et al. Evaluation of circulating cathodic antigen (CCA) urine-cassette assay as a survey tool for Schistosoma mansoni in different transmission settings within Bugiri District, Uganda. Acta Trop. 2014; 136: 50-7.

Becker SL, Marti H, Zimmermann S, Vidacek D, Herrmann M, Utzinger J, et al. Application in Europe of a urine-based rapid diagnostic test for confirmation of Schistosoma mansoni infection in migrants from endemic areas. Euro Surveill. 2015; 20(23): 9-13.

CDC - Centers for Disease Control and Prevention. Parasites-Schistosomiasis. 2016. Available from: http://www.cdc.gov/parasites/ schistosomiasis/health_professionals/index.html\#dx.

Clerinx J, van Gompel A. Schistosomiasis in travelers and migrants. Travel Med Infect Dis. 2011; 9(1): 6-24.

Colley DG, Binder S, Campbell C, King CH, Tchuente LA, N'Goran $\mathrm{EK}$, et al. A five-country evaluation of a point-of-care circulating cathodic antigen urine assay for the prevalence of Schistosoma mansoni. Am J Trop Med Hyg. 2013; 88(3): 426-32.

Coulibaly JT, Knopp S, N'Guessan NA, Silué KD, Fürst T, Lohourignon LK, et al. Accuracy of urine circulating cathodic antigen (CCA) test for Schistosoma mansoni diagnosis in different settings of Côte d'Ivoire. PLoS Negl Trop Dis. 2011; 5(11): e1384. 
Coulibaly JT, N'Gbesso YK, Knopp S, N'Guessan NA, Silué KD, van Dam GJ, et al. Accuracy of urine circulating cathodic antigen test for the diagnosis of Schistosoma mansoni in preschool-aged children before and after treatment. PLoS Negl Trop Dis. 2013; 7(3): e2109.

de Laval F, Savini H, Biance-Valero E, Simon F. Human schistosomiasis: an emerging threat for Europe. Lancet. 2014; 384(9948): 1094-5.

Erko B, Medhin G, Teklehaymanot T, Degarege A, Legesse M. Evaluation of urine-circulating cathodic antigen (Urine-CCA) cassette test for the detection of Schistosoma mansoni infection in areas of moderate prevalence in Ethiopia. Trop Med Int Health. 2013; 18(8): 1029-35.

Gomes LI, Enk MJ, Rabello A. Diagnosing schistosomiasis: where are we? Rev Soc Bras Med Trop. 2014; 47(1): 3-11.

Hotez PJ, Alvarado M, Basanez MG, Basáñez MG, Bolliger I, Bourne R, et al. The global burden of disease study 2010: interpretation and implications for the neglected tropical diseases. PLoS Negl Trop Dis. 2014; 8(7): e2865.

Katz N, Chaves A, Pellegrino J. A simple device for quantitative stool thick-smear technique in schistosomiasis mansoni. Rev Inst Med Trop Sao Paulo. 1972; 14(6): 397-400.

Midzi N, Butterworth AE, Mduluza T, Munyati S, Deelder AM, van Dam GJ. Use of circulating cathodic antigen strips for the diagnosis of urinary schistosomiasis. Trans R Soc Trop Med Hyg. 2009; 103(1): 45-51.

Navaratnam AMD, Mutumba-Nakalembea MJ, Stothardb JR, Kabatereinec NB, Fenwicka A, Sousa-Figueiredob JC. Notes on the use of urine-CCA dipsticks for detection of intestinal schistosomiasis in preschool children. Trans R Soc Trop Med Hyg. 2012; 106(10): 619-22.

Obeng BB, Aryeetey YA, de Dood CJ, Amoah AS, Larbi IA, Deelder $\mathrm{AM}$, et al. Application of a circulating-cathodic-antigen (CCA) strip test and real-time PCR, in comparison with microscopy, for the detection of Schistosoma haematobium in urine samples from Ghana. Ann Trop Med Parasitol. 2008; 102(7): 625-33.

Ochodo EA, Gopalakrishna G, Spek B, Reitsma JB, van Lieshout $\mathrm{L}$, Polman K, et al. Circulating antigen tests and urine reagent strips for diagnosis of active schistosomiasis in endemic areas (Review). Cochrane Database Syst Rev. 2015; 11(3): 1-292.

Ruganuza DM, Mazigo HD, Waihenya R, Morona D, Mkoji GM. Schistosoma mansoni among pre-school children in Musozi village, Ukerewe Island, North-Western-Tanzania: prevalence and associated risk factors. Parasit Vectors. 2015; 16(8): 377.

Shane HL, Verani JR, Abudho B, Montgomery SP, Blackstock AJ, Mwinzi PN, et al. Evaluation of urine CCA assays for detection of Schistosoma mansoni infection in Western Kenya. PLoS Negl Trop Dis. 2011; 5(1): e951.

Sousa-Figueiredo JC, Betson M, Kabatereine NB, Stothard JR. The urine circulating cathodic antigen (CCA) dipstick: a valid substitute for microscopy for mapping and point-of-care diagnosis of intestinal schistosomiasis. PLoS Negl Trop Dis. 2013; 7(1): e2008.

Stothard JR, Sousa-Figueiredo JC, Betson M, Adriko M, Arinaitwe M, Rowell C, et al. Schistosoma mansoni infections in young children: when are schistosome antigens in urine, eggs in stool and antibodies to eggs first detectable? PLoS Negl Trop Dis. 2011; 5(1): e938.

Stothard JR, Sousa-Figueiredo JC, Standley C, Van Dam GJ, Knopp S, Utzinger J, et al. An evaluation of urine-CCA strip test and fingerprick blood SEA-ELISA for detection of urinary schistosomiasis in schoolchildren in Zanzibar. Acta Trop. 2009; 111(1): 64-70.

Tchuenté LAT, Fouodo CJK, Ngassam RIK, Sumo L, Noumedem CD, Kenfack CM, et al. Evaluation of circulating cathodic antigen (CCA) urine-tests for diagnosis of Schistosoma mansoni infection in Cameroon. PLoS Negl Trop Dis. 2012; 6(7): e1758.

Utzinger J, Becker SL, Knopp S, Blum J, Neumayr AL, Keiser J, et al. Neglected tropical diseases: diagnosis, clinical management, treatment and control. Swiss Med Wkly. 2012; 142: 24. 